\title{
Heterogeneity in national U.S. mortality trends within heart disease subgroups, 2000-2015
}

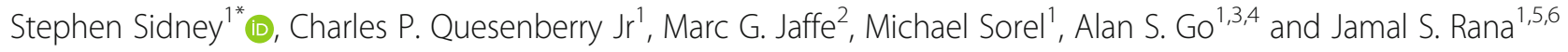

\begin{abstract}
Background: The long-term downward national U.S. trend in heart disease-related mortality slowed substantially during 2011-2014 before turning upward in 2015. Examining mortality trends in the major subgroups of heart disease may provide insight into potentially more targeted and effective prevention and treatment approaches to promote favorable trajectories. We examined national trends between 2000 and 2015 in mortality attributed to major heart disease subgroups including ischemic heart disease, heart failure, and all other types of heart disease.
\end{abstract}

Methods: Using the Centers for Disease Control and Prevention Wide-ranging Online Data for Epidemiologic Research (WONDER) data system, we determined national trends in age-standardized mortality rates attributed to ischemic heart disease, heart failure, and other heart diseases from January 1, 2000, to December 31, 2011, and from January 1, 2011, to December 31, 2015. Annual rate of changes in mortality attributed to ischemic heart disease, heart failure, and other heart diseases for 2000-2011 and 2011-2015 were compared.

Results: Death attributed to ischemic heart disease declined from 2000 to 2015, but the rate of decline slowed from 4.96\% (95\% confidence interval 4.77\%-5.15\%) for 2000-2011 to 2.66\% (2.00\%-3.31\%) for 2011-2015. In contrast, death attributed to heart failure and all other causes of heart disease declined from 2000 to 2011 at annual rates of $1.94 \%(1.77 \%-2.11 \%)$ and $0.64 \%(0.44 \%-0.82 \%)$ respectively, but increased from 2011 to 2015 at annual rates of 3.73\% (3.21\% 4.26\%) and 1.89\% (1.33-2.46\%). Differences in 2000-2011 and 2011-2015 decline rates were statistically significant for all 3 endpoints overall, by sex, and all race/ethnicity groups except Asian/Pacific Islanders (heart failure only significant) and American Indian/Alaskan Natives.

Conclusions: While the long-term decline in death attributed to heart disease slowed between 2011 and 2014 nationally before turning upward in 2015, heterogeneity existed in the trajectories attributed to heart disease subgroups, with ischemic heart disease mortality continuing to decline while death attributed to heart failure and other heart diseases switched from a downward to upward trend. While systematic efforts to prevent and treat ischemic heart disease continue to be effective, urgent attention is needed to address the challenge of heart failure.

Keywords: Mortality rate, Heart disease, Coronary heart disease, Heart failure, Epidemiology

\section{Background}

We recently reported that the rate of decline of death attributed to total cardiovascular disease (CVD) and to heart disease (HD) in the U.S. had decelerated substantially between 2011 and 2014 [1], with the annualized percent decline in CVD and HD mortality decreasing

\footnotetext{
* Correspondence: Steve.Sidney@kp.org

'Division of Research, Kaiser Permanente Northern California, 2000 Broadway, Oakland, CA 94612, USA

Full list of author information is available at the end of the article
}

from $3.79 \%$ and $3.69 \%$ respectively for $2000-2011$ to $0.65 \%$ and $0.76 \%$ for $2011-2014$. We suggested that HD mortality might increase in 2015 [1] which was confirmed by the recent report of a $0.9 \%$ increase from 167.0 to 168.5 per 100,000 person-years from 2014 to 2015 , the first year-to-year increase since 1992-93 [2, 3].

HD-related death encompasses a wide range of heart conditions. Thus, from both prevention and intervention perspectives, it is important to further delineate trends in subcategories of HD-related death. We studied 
mortality trends in the two largest subgroups of HD (ischemic heart disease [IHD] and heart failure [HF]) and in all other HD combined.

\section{Methods}

Mortality rates between 2000 and 2015 were ascertained using the U.S. Centers for Disease Control and Prevention's Wide-Ranging Online Data for Epidemiologic Research (CDC WONDER) dataset, which includes the assigned cause of death from all death certificates filed in the 50 states and the District of Columbia [3]. Categorization of the presumed cause of death used International Statistical Classification of Diseases and Related Health Problems, Tenth Edition codes as follows: HD (codes I00-I09, I11, I13, and I20-I51), IHD (I20-I25), HF (I50), and all other causes of HD (I00-I09, I11, I13, I26-I49, and I51).

This study did not require institutional review board approval because it analyzes government-issued public use data without individual identifiable information.

Age-standardized mortality rates (AAMR) were calculated using the direct method, with the 2000 U.S. Census as the standard population using the following age categorization: younger than 1 year, 1 to 4,5 to 14,15 to 24, 25 to 34,35 to 44,45 to 54,55 to 64,65 to 74,75 to 84 , and 85 years or older [4]. Poisson regression with allowance for overdispersion was used for point and interval estimation of age-adjusted annual rates of change for
January 1, 2000, to December 31, 2011, and January 1, 2011, to December 31, 2015.

\section{Results}

Mortality rates from 2000 to 2015 for HD and HD subgroups are shown in Table 1, with the largest subgroup being IHD. Compared to 2014, in 2015, an increase in overall HD occurred in men (0.4\%), women (1.4\%), and in all racial-ethnic groups except $\mathrm{NH}$ Blacks in which HD mortality decreased by $0.3 \%$. The 2015 mortality rate for each HD subgroup was higher in men than in women. By race-ethnicity, $\mathrm{NH}$ blacks had the highest mortality rate for each HD subcategory, followed by $\mathrm{NH}$ whites, NH American Indian/Alaskan Natives, Hispanics, and NH Asian/Pacific Islanders.

The rate of decline in death attributed to IHD slowed in 2011-2015, with mean annual rate of change of $-2.66 \%$ compared to $-4.96 \%$ for $2000-2011$ (Table 2, Fig. 1). The difference in the rate of change between the two time periods was statistically significant overall, in each sex and, among $\mathrm{NH}$ whites, $\mathrm{NH}$ blacks, and Hispanics.

In sharp contrast, mortality rates attributed to HF and all other HD declined from 2000 to 2011, but then increased from 2011 to 2015 (Table 2, Fig. 1). These patterns were evident in both sexes (Tables 3 and 4 and Fig. 1) and in all race-ethnicity groups except NH American Indian/Alaskan Natives (Tables 5,

Table 1 Age-adjusted mortality rates for all heart disease, ischemic heart disease, heart failure, and all other CHD, United States, 2000-2015

\begin{tabular}{|c|c|c|c|c|c|c|c|c|c|}
\hline \multirow[b]{2}{*}{ Year } & \multirow[b]{2}{*}{$N=$ Population } & \multicolumn{2}{|c|}{ Heart disease } & \multicolumn{2}{|c|}{ Ischemic HD } & \multicolumn{2}{|l|}{ Heart failure } & \multicolumn{2}{|l|}{ All other HD } \\
\hline & & $\mathrm{n}=$ deaths & $\overline{\text { AAMR }^{a}}$ & $\mathrm{n}=$ deaths & $\overline{A A M R}$ & $\mathrm{n}=$ deaths & $\overline{A A M R}$ & $\mathrm{n}=$ deaths & $\overline{A A M R}$ \\
\hline 2000 & $281,421,906$ & 710,760 & 257.6 & 515,204 & 186.8 & 55,704 & 20.3 & 139,852 & 50.6 \\
\hline 2001 & $284,968,955$ & 700,142 & 249.5 & 502,189 & 179.0 & 56,934 & 20.4 & 141,019 & 50.2 \\
\hline 2002 & $287,625,193$ & 696,947 & 244.6 & 494,382 & 173.5 & 56,494 & 19.9 & 146,071 & 51.2 \\
\hline 2003 & $290,107,933$ & 685,089 & 236.3 & 480,028 & 165.6 & 57,448 & 19.9 & 147,613 & 50.9 \\
\hline 2004 & $292,805,298$ & 652,486 & 221.6 & 451,326 & 153.2 & 57,120 & 19.5 & 144,040 & 48.9 \\
\hline 2005 & $295,516,599$ & 652,091 & 216.8 & 445,687 & 148.2 & 58,933 & 19.7 & 147,471 & 49.0 \\
\hline 2006 & $298,379,912$ & 631,636 & 205.5 & 425,425 & 138.3 & 60,337 & 19.7 & 145,874 & 47.5 \\
\hline 2007 & $301,231,207$ & 616,067 & 196.1 & 406,351 & 129.2 & 56,565 & 18.0 & 153,151 & 48.8 \\
\hline 2008 & $304,093,966$ & 616,828 & 192.1 & 405,309 & 126.1 & 56,830 & 17.7 & 154,689 & 48.3 \\
\hline 2009 & $306,771,529$ & 599,413 & 182.8 & 386,324 & 117.7 & 56,410 & 17.2 & 156,679 & 47.9 \\
\hline 2010 & $308,745,538$ & 597,689 & 179.1 & 379,559 & 113.6 & 57,757 & 17.3 & 160,373 & 48.2 \\
\hline 2011 & $311,591,917$ & 596,577 & 173.7 & 375,295 & 109.2 & 58,309 & 16.9 & 162,973 & 47.7 \\
\hline 2012 & $313,914,040$ & 599,711 & 170.5 & 371,469 & 105.4 & 60,341 & 17.1 & 167,901 & 48.0 \\
\hline 2013 & $316,128,839$ & 611,105 & 169.8 & 370,213 & 102.6 & 65,120 & 18.0 & 175,772 & 49.1 \\
\hline 2014 & $318,857,056$ & 614,348 & 167.0 & 364,593 & 98.8 & 68,626 & 18.6 & 181,129 & 49.6 \\
\hline 2015 & $321,418,820$ & 633,842 & 168.5 & 366,801 & 97.2 & 75,251 & 19.9 & 191,790 & 51.4 \\
\hline
\end{tabular}

Abbreviations: $H D$ heart disease, $A A M R$ age-adjusted mortality rate

${ }^{a}$ Age-adjusted mortality rate per 100,000 person-years, directly standardized to the 2000 U.S. population 
Table 2 Age-adjusted mortality rates and annual rates of change for ischemic heart disease, heart failure, and other heart disease for time periods 2000-2011 and 2011-2015, United States

\begin{tabular}{|c|c|c|c|c|c|c|}
\hline \multirow[b]{2}{*}{ Year(s) } & \multicolumn{3}{|c|}{ AAMR } & \multicolumn{3}{|c|}{ Annual rate of change $(\%)^{a}$} \\
\hline & 2000 & 2011 & 2015 & $2000-2011$ & $2011-2015$ & $p$-value ${ }^{b}$ \\
\hline \multicolumn{7}{|c|}{ Ischemic heart disease } \\
\hline Total & 186.8 & 109.2 & 97.2 & $-4.96(-5.15$ to -4.77$)$ & $-2.66(-3.31$ to -2.00$)$ & $<0.001$ \\
\hline Total male & 241.4 & 145.6 & 131.2 & $-4.63(-4.82$ to -4.44$)$ & $-2.10(-2.75$ to -1.45$)$ & $<0.001$ \\
\hline Total female & 146.5 & 81.0 & 70.5 & $-5.49(-5.69$ to -5.29$)$ & $-3.69(-4.29$ to -2.88$)$ & $<0.001$ \\
\hline $\mathrm{NH}$ White & 186.6 & 111.1 & 99.7 & $-4.85(-5.05$ to -4.64$)$ & $-2.34(-3.05$ to -1.63$)$ & $<0.001$ \\
\hline NH Asian/PI & 109.7 & 63,2 & 55.1 & $-4.71(-5.03$ to -4.43$)$ & $-3.75(-4.64$ to -2.85$)$ & 0.08 \\
\hline Hispanic & 153.2 & 84.2 & 74.5 & $-5.38(-5.64$ to -5.12$)$ & $-3.39(-4.21$ to -2.58$)$ & $<0.001$ \\
\hline NH Black & 220.4 & 127.9 & 111.3 & $-5.06(-5.26$ to -4.86$)$ & $-3.16(-3.93$ to -2.49$)$ & 0.003 \\
\hline $\mathrm{NH}$ Al/AN & 142.7 & 104.8 & 95.2 & $-3.04(-3.55$ to -2.52$)$ & $-1.23(-2.79$ to 0.36$)$ & 0.06 \\
\hline \multicolumn{7}{|l|}{ Heart failure } \\
\hline Total & 20.3 & 16.9 & 19.9 & $-1.94(-2.11$ to -1.77$)$ & 3.73 (3.21 to 4.26$)$ & $<0.001$ \\
\hline Total male & 21.5 & 18.7 & 22.5 & $-1.51(-1.70$ to -1.31$)$ & 4.58 (4.00 to 5.17$)$ & $<0.001$ \\
\hline Total female & 19.2 & 15.6 & 17.9 & $-2.26(-2.44$ to -2.09$)$ & 2.99 (2.43 to 3.56$)$ & $<0.001$ \\
\hline NH White & 20.7 & 17.5 & 20.8 & $-1.86(-2.02$ to -1.70$)$ & 4.10 (3.60 to 4.61$)$ & $<0.001$ \\
\hline $\mathrm{NH}$ Asian/PI & 7.8 & 6.4 & 7.3 & $-0.95(-1.68$ to -0.22$)$ & 4.14 (2.28 to 6.04$)$ & $<0.001$ \\
\hline Hispanic & 10.9 & 10.7 & 11.3 & $-0.94(-1.40$ to -0.48$)$ & 1.87 (0.65 to 3.11$)$ & $<0.001$ \\
\hline NH Black & 22.4 & 19.1 & 23.3 & $-1.66(-1.98$ to -1.34$)$ & 4.40 (3.43 to 5.37$)$ & $<0.001$ \\
\hline $\mathrm{NH} \mathrm{Al/AN}$ & 16.7 & 14.9 & 15.0 & $-1.12(-2.14$ to -0.09$)$ & $-2.08(-4.95$ to 0.89$)$ & 0.60 \\
\hline \multicolumn{7}{|c|}{ Other heart disease } \\
\hline Total & 50.6 & 47.7 & 51.4 & $-0.63(-0.82$ to -0.44$)$ & 1.89 (1.33 to 2.46$)$ & $<0.001$ \\
\hline Total male & 57.1 & 53.9 & 58.1 & $-0.59(-0.79$ to 0.39$)$ & 2.04 (1.45 to 2.63$)$ & $<0.001$ \\
\hline Total female & 45.1 & 42.1 & 45.2 & $-0.69(-0.90$ to -0.49$)$ & 1.73 (1.11 to 2.36$)$ & $<0.001$ \\
\hline NH White & 48.1 & 47.1 & 51.5 & $-0.34(-0.55$ to -0.22$)$ & $2.52(1.87$ to 3.17$)$ & $<0.001$ \\
\hline NH Asian/PI & 28.6 & 24.2 & 24.2 & $-1.70(-2.06$ to -1.33$)$ & $-0.38(-1.37$ to 0.61$)$ & 0.04 \\
\hline Hispanic & 31.9 & 31.4 & 31.9 & $-0.93(-1.19$ to -0.66$)$ & $0.78(-0.06$ to 1.51$)$ & $<0.001$ \\
\hline NH Black & 85.7 & 72.1 & 75.5 & $-1.51(-1.67$ to -1.35$)$ & $0.42(-0.06$ to 0.91$)$ & $<0.001$ \\
\hline $\mathrm{NH}$ Al/AN & 38.5 & 41.3 & 44.8 & $0.55(-0.24$ to 1.34$)$ & 2.57 (0.41 to 4.78$)$ & 0.14 \\
\hline
\end{tabular}

Abbreviations: AAMR age-adjusted mortality rate, $\mathrm{NH}$ non-Hispanic, PI Pacific Islander, Al/AN American Indian/Alaskan Native

${ }^{a}$ Annual rate of change age-adjusted by Poisson regression

${ }^{\mathrm{b}} p$-value for difference in annual rate of change between 2000 and 2011 and 2011-2015 time periods

6, 7, 8 and 9, Fig. 1). From 2011 to 2015, the mean annual rate of increase was $3.73 \%$ for HF-related mortality and $1.89 \%$ for all other HD mortality in the total population. The difference in the rate of change between the two time periods was statistically significant overall in each sex, and in all race-ethnicity groups except $\mathrm{NH}$ American Indian/Alaskan Natives for HF and other HD mortality as well as NH Asian/ Pacific Islander for other HD mortality (Table 2). Trends in crude mortality rates (Table 10) for HD and each HD subgroup were similar to age-standardized mortality trends.

Five specific ICD-10 codes accounted for $63 \%$ of deaths attributed to other HD during 2011-2015. There was an increase in age-standardized mortality rates per 100,000 person-years from 9.7 to 11.1 for hypertensive HD (ICD-10 code I11), 5.2 to $6.3(p<0.001)$ for atrial fibrillation and flutter (ICD-10 code I48), and a decrease from 6.8 to $6.3(p<0.001)$ for cardiomyopathy (I42). Changes were not statistically significant for nonrheumatic aortic valve disorders (I35), 4.5 to $4.6(p=0.45)$; and cardiac arrest (I46), 4.4 to $4.3(p=0.48)$.

\section{Discussion}

The increase in death attributed to HD in 2015 represents a notable landmark denoting a time where the impact of prevention efforts has been at least temporarily stalled. HD mortality increased across both sexes and 

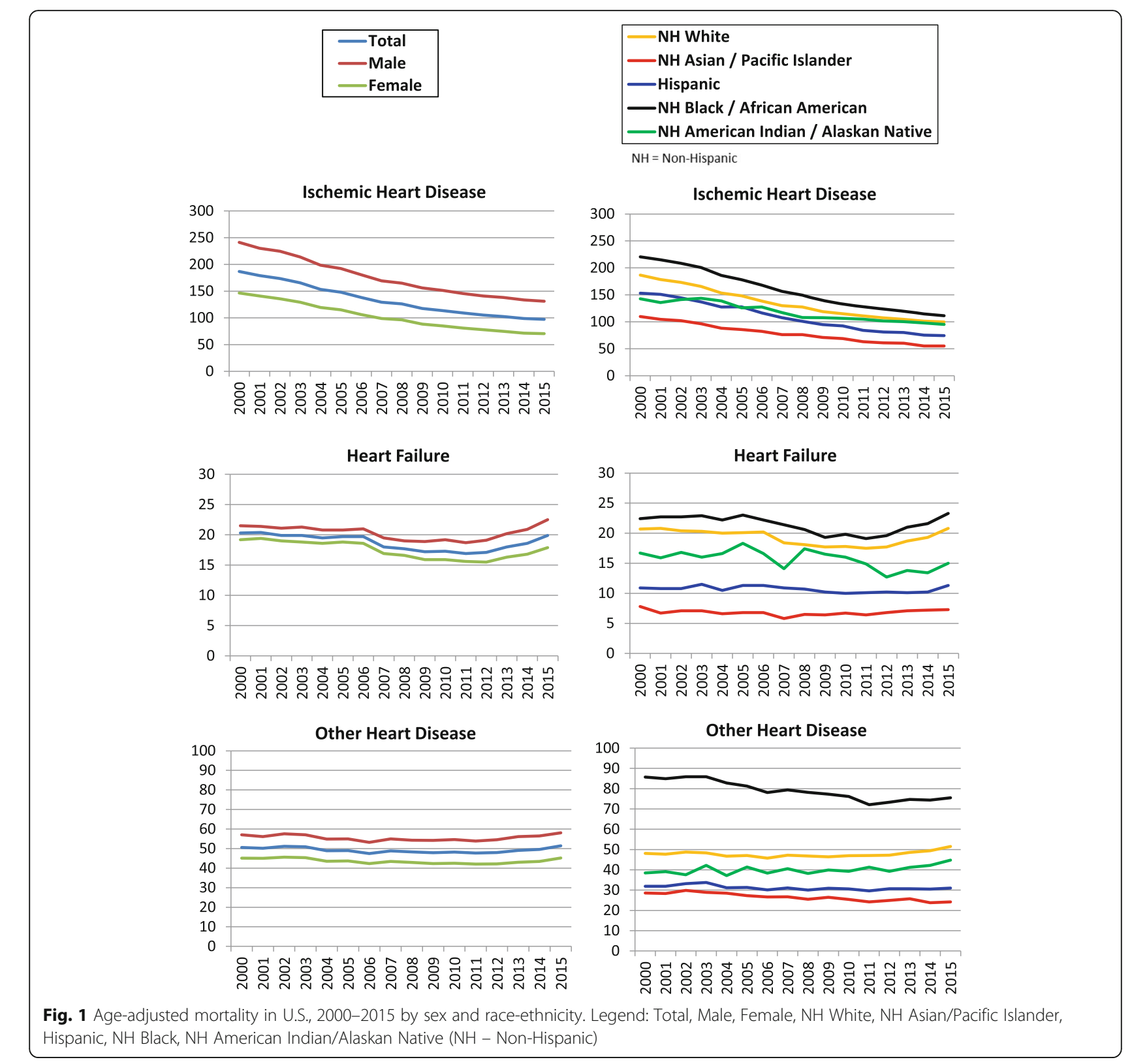

most race-ethnicity groups. Although a slight decline was noted for $\mathrm{NH}$ blacks, HD-related death rates in this subgroup remain substantially higher than in other racial/ethnic groups.

While the continued decline in IHD mortality is encouraging, the rate of decline decreased by nearly $50 \%$ during the 2011-2015 period compared to 2000-2011. The decades-long epidemic of obesity and diabetes mellitus are likely important factors contributing the deceleration of the rate of decline of cardiovascular mortality nationally [1]. A recent study analyzing data from several cohort studies demonstrated a substantial decrease in the incidence of new-onset IHD between two time periods, with baseline exams conducted from 1983 to 1990 and 1996 to 2001, and showed that the fraction of CHD attributable to diabetes decreased over time [5]. However, the prevalence of diabetes has risen considerably from the time period that diabetes was assessed for these studies, [6] and populations now living with longer duration of diabetes have higher risk of CHD [7]. Additionally, follow-up ended in 2011, the year that the IHD mortality trend change occurred, so that the findings regarding the decreasing fraction of CHD attributable to diabetes are likely to not be as relevant to the current time period.

Several U.S.-based studies have shown decline in the incidence of acute myocardial infarction with follow-up through 2008-2011, [8-11] with one reporting additional 
Table 3 Males (age-adjusted)

\begin{tabular}{|c|c|c|c|c|c|c|c|c|c|}
\hline \multirow[b]{2}{*}{ Year } & \multirow[b]{2}{*}{ ( $n=$ Population) } & \multicolumn{2}{|l|}{ Heart disease } & \multicolumn{2}{|l|}{ Ischemic HD } & \multicolumn{2}{|l|}{ Heart failure } & \multicolumn{2}{|l|}{ All other HD } \\
\hline & & ( $\mathrm{n}=$ deaths) & AAMR & ( $\mathrm{n}=$ deaths) & AAMR & ( $\mathrm{n}=$ deaths) & AAMR & ( $\mathrm{n}=$ deaths) & AAMR \\
\hline 2000 & $138,053,563$ & 344,807 & 320.0 & 260,574 & 241.4 & 21,175 & 21.5 & 63,058 & 57.1 \\
\hline 2001 & $139,891,492$ & 339,095 & 307.8 & 254,005 & 230.2 & 21,632 & 21.4 & 63,458 & 56.1 \\
\hline 2002 & $141,230,559$ & 340,933 & 303.4 & 252,760 & 224.7 & 21,698 & 21.1 & 66,475 & 57.6 \\
\hline 2003 & $142,428,897$ & 336,095 & 292.3 & 246,342 & 213.9 & 22,427 & 21.3 & 67,326 & 57.1 \\
\hline 2004 & $143,828,012$ & 321,973 & 274.1 & 233,538 & 198.4 & 22,292 & 20.8 & 66,143 & 54.9 \\
\hline 2005 & $145,197,078$ & 322,841 & 268.2 & 232,115 & 192.3 & 23,026 & 20.8 & 67,700 & 55.0 \\
\hline 2006 & $146,647,265$ & 315,706 & 254.9 & 224,510 & 180.7 & 23,918 & 21.0 & 67,278 & 53.2 \\
\hline 2007 & $148,064,854$ & 309,821 & 243.7 & 216,050 & 169.2 & 22,914 & 19.5 & 70,857 & 55.0 \\
\hline 2008 & $149,489,951$ & 311,201 & 238.5 & 216,248 & 165.1 & 23,017 & 19.0 & 71,936 & 54.3 \\
\hline 2009 & $150,807,454$ & 307,225 & 229.4 & 210,069 & 156.2 & 23,563 & 18.9 & 73,593 & 54.2 \\
\hline 2010 & $151,781,326$ & 307,384 & 225.1 & 207,580 & 151.3 & 24,385 & 19.2 & 75,419 & 54.6 \\
\hline 2011 & $153,290,819$ & 308,398 & 218.1 & 206,908 & 145.6 & 24,609 & 18.7 & 76,881 & 53.9 \\
\hline 2012 & $154,492,067$ & 312,491 & 214.7 & 206,685 & 141.1 & 26,036 & 19.1 & 79,770 & 54.5 \\
\hline 2013 & $155,651,602$ & 321,347 & 214.5 & 208,515 & 138.2 & 28,513 & 20.2 & 84,319 & 56.1 \\
\hline 2014 & $156,936,487$ & 325,077 & 210.9 & 207,412 & 133.5 & 30,339 & 20.9 & 87,326 & 56.5 \\
\hline 2015 & $158,229,297$ & 335,002 & 211.8 & 209,298 & 131.2 & 33,667 & 22.5 & 92,037 & 58.1 \\
\hline
\end{tabular}

Age-adjusted mortalilty rate per 100,000 person-years, directly standardized to the 2000 U.S. population

Table 4 Female (age-adjusted)

\begin{tabular}{|c|c|c|c|c|c|c|c|c|c|}
\hline \multicolumn{10}{|c|}{ Trends in mortality in United States from 2000 to 2015 by gender and race-ethnicity } \\
\hline \multirow[b]{2}{*}{ Year } & \multirow[b]{2}{*}{ ( $n=$ Population) } & \multicolumn{2}{|l|}{ Heart disease } & \multicolumn{2}{|l|}{ Ischemic HD } & \multicolumn{2}{|l|}{ Heart failure } & \multicolumn{2}{|l|}{ All other HD } \\
\hline & & $(\mathrm{n}=$ deaths $)$ & AAMR & $(\mathrm{n}=$ deaths $)$ & AAMR & $(\mathrm{n}=$ deaths $)$ & AAMR & $(n=$ deaths $)$ & AAMR \\
\hline 2000 & $143,368,343$ & 365,953 & 210.9 & 254,630 & 146.5 & 34,529 & 19.2 & 76,794 & 45.1 \\
\hline 2001 & $145,077,463$ & 361,047 & 205.4 & 248,184 & 140.9 & 35,302 & 19.4 & 77,561 & 45.0 \\
\hline 2002 & $146,394,634$ & 356,014 & 200.3 & 241,622 & 135.7 & 34,796 & 19.0 & 79,596 & 45.6 \\
\hline 2003 & $147,679,036$ & 348,994 & 193.7 & 233,686 & 129.4 & 35,021 & 18.8 & 80,287 & 45.4 \\
\hline 2004 & $148,977,286$ & 330,513 & 181.5 & 217,788 & 119.4 & 34,828 & 18.6 & 77,897 & 43.5 \\
\hline 2005 & $150,319,521$ & 329,250 & 177.5 & 213,572 & 115.0 & 35,907 & 18.8 & 79,771 & 43.7 \\
\hline 2006 & $151,732,647$ & 315,930 & 167.2 & 200,915 & 106.3 & 36,419 & 18.6 & 78,596 & 42.3 \\
\hline 2007 & $153,166,353$ & 306,246 & 159.0 & 190,301 & 98.8 & 33,651 & 16.9 & 82,294 & 43.4 \\
\hline 2008 & $154,604,015$ & 305,627 & 155.9 & 189,061 & 96.3 & 33,813 & 16.6 & 82,753 & 42.9 \\
\hline 2009 & $155,964,075$ & 292,188 & 146.6 & 176,255 & 88.4 & 32,847 & 15.9 & 83,086 & 42.3 \\
\hline 2010 & $156,964,212$ & 290,305 & 143.3 & 171,979 & 84.9 & 33,372 & 15.9 & 84,954 & 42.5 \\
\hline 2011 & $158,301,098$ & 288,179 & 138.7 & 168,387 & 81.0 & 33,700 & 15.6 & 86,092 & 42.1 \\
\hline 2012 & $159,421,973$ & 287,220 & 135.5 & 164,784 & 77.8 & 34,305 & 15.5 & 88,131 & 42.2 \\
\hline 2013 & $160,477,237$ & 289,758 & 134.3 & 161,698 & 74.9 & 36,607 & 16.3 & 91,453 & 43.0 \\
\hline 2014 & $161,920,569$ & 289,271 & 131.8 & 157,181 & 71.6 & 38,287 & 16.8 & 93,803 & 43.4 \\
\hline 2015 & $163,189,523$ & 298,840 & 133.6 & 157,503 & 70.5 & 41,584 & 17.9 & 99,753 & 45.2 \\
\hline
\end{tabular}


Table 5 Non-Hispanic White (age-adjusted)

\begin{tabular}{|c|c|c|c|c|c|c|c|c|c|}
\hline \multirow[b]{2}{*}{ Year } & \multirow[b]{2}{*}{ ( $n=$ Population) } & \multicolumn{2}{|l|}{ Heart disease } & \multicolumn{2}{|l|}{ Ischemic HD } & \multicolumn{2}{|l|}{ Heart failure } & \multicolumn{2}{|l|}{ All other HD } \\
\hline & & ( $\mathrm{n}=$ deaths) & AAMR & ( $\mathrm{n}=$ deaths) & AAMR & ( $\mathrm{n}=$ deaths) & AAMR & ( $\mathrm{n}=$ deaths) & AAMR \\
\hline 2000 & $197,324,684$ & 594,465 & 255.5 & 434,505 & 186.6 & 48,782 & 20.7 & 111,178 & 48.1 \\
\hline 2001 & $197,842,671$ & 582,349 & 247.2 & 420,959 & 178.5 & 49,788 & 20.8 & 111,602 & 47.7 \\
\hline 2002 & $198,101,982$ & 577,761 & 242.5 & 413,230 & 173.2 & 49,162 & 20.4 & 115,369 & 48.8 \\
\hline 2003 & $198,289,486$ & 565,808 & 234.2 & 400,101 & 165.5 & 49,788 & 20.3 & 115,919 & 48.4 \\
\hline 2004 & $198,619,903$ & 537,512 & 220.1 & 374,900 & 153.3 & 49,628 & 20 & 112,984 & 46.7 \\
\hline 2005 & $198,880,984$ & 535,101 & 215.5 & 368,505 & 148.3 & 50,835 & 20.1 & 115,761 & 47.1 \\
\hline 2006 & $199,200,396$ & 516,883 & 204.5 & 350,356 & 138.6 & 52,125 & 20.2 & 114,402 & 45.8 \\
\hline 2007 & $199,492,421$ & 502,683 & 195.5 & 334,047 & 129.9 & 48,480 & 18.4 & 120,156 & 47.2 \\
\hline 2008 & $199,783,797$ & 503,096 & 192.4 & 333,378 & 127.4 & 48,518 & 18.1 & 121,200 & 46.8 \\
\hline 2009 & $199,993,079$ & 485,779 & 182.9 & 315,810 & 118.9 & 48,156 & 17.7 & 121,813 & 46.4 \\
\hline 2010 & $200,127,372$ & 483,973 & 179.9 & 309,492 & 115.0 & 49,253 & 17.8 & 125,228 & 47.0 \\
\hline 2011 & $200,423,243$ & 482,979 & 175.6 & 305,486 & 111.1 & 49,605 & 17.5 & 127,888 & 47.1 \\
\hline 2012 & $200,698,847$ & 481,991 & 172.3 & 300,439 & 107.4 & 50,922 & 17.7 & 130,630 & 47.2 \\
\hline 2013 & $200,918,513$ & 488,817 & 171.8 & 297,501 & 104.6 & 54,787 & 18.7 & 136,529 & 48.6 \\
\hline 2014 & $201,048,793$ & 489,926 & 169.9 & 291,879 & 101.2 & 57,522 & 19.3 & 140,525 & 49.4 \\
\hline 2015 & $201,242,281$ & 503,172 & 171.9 & 291,850 & 99.7 & 62,649 & 20.8 & 148,673 & 51.5 \\
\hline
\end{tabular}

Age-adjusted mortalilty rate per 100,000 person-years, directly standardized to the 2000 U.S. population

Table 6 Non-Hispanic Asian/Pacific Islander (age-adjusted)

\begin{tabular}{|c|c|c|c|c|c|c|c|c|c|}
\hline \multicolumn{10}{|c|}{ Trends in mortality in United States from 2000 to 2015 by gender and race-ethnicity } \\
\hline \multirow[b]{2}{*}{ Year } & \multirow[b]{2}{*}{ ( $n=$ Population) } & \multicolumn{2}{|l|}{ Heart disease } & \multicolumn{2}{|l|}{ Ischemic HD } & \multicolumn{2}{|l|}{ Heart failure } & \multicolumn{2}{|l|}{ All other HD } \\
\hline & & $(\mathrm{n}=$ deaths $)$ & AAMR & $(\mathrm{n}=$ deaths $)$ & AAMR & $(\mathrm{n}=$ deaths $)$ & AAMR & $(n=$ deaths $)$ & AAMR \\
\hline 2000 & $11,355,553$ & 8949 & 146.1 & 6689 & 109.7 & 418 & 7.8 & 1842 & 28.6 \\
\hline 2001 & $11,983,178$ & 9291 & 139.5 & 6916 & 104.5 & 392 & 6.7 & 1983 & 28.3 \\
\hline 2002 & $12,472,384$ & 9814 & 139.2 & 7159 & 102.3 & 445 & 7.1 & 2210 & 29.9 \\
\hline 2003 & $12,942,337$ & 9934 & 132.5 & 7221 & 96.5 & 474 & 7.1 & 2239 & 28.9 \\
\hline 2004 & $13,406,530$ & 9756 & 123.4 & 6954 & 88.2 & 475 & 6.6 & 2327 & 28.5 \\
\hline 2005 & $13,888,295$ & 10,281 & 119.8 & 7329 & 85.7 & 519 & 6.8 & 2433 & 27.3 \\
\hline 2006 & $14,375,996$ & 10,457 & 115.7 & 7430 & 82.3 & 556 & 6.8 & 2471 & 26.6 \\
\hline 2007 & $14,854,701$ & 10,394 & 108.6 & 7292 & 76.1 & 504 & 5.8 & 2598 & 26.7 \\
\hline 2008 & $15,336,181$ & 10,951 & 108.1 & 7705 & 76.1 & 606 & 6.5 & 2640 & 25.5 \\
\hline 2009 & $15,793,995$ & 11,134 & 103.8 & 7616 & 70.9 & 638 & 6.4 & 2880 & 26.5 \\
\hline 2010 & $16,133,872$ & 11,254 & 101.1 & 7683 & 69 & 694 & 6.7 & 2877 & 25.4 \\
\hline 2011 & $16,579,709$ & 11,406 & 93.8 & 7712 & 63.2 & 714 & 6.4 & 2980 & 24.2 \\
\hline 2012 & $17,175,596$ & 12,068 & 92.7 & 7959 & 61 & 825 & 6.8 & 3284 & 24.9 \\
\hline 2013 & $17,693,870$ & 13,064 & 93.2 & 8477 & 60.3 & 954 & 7.1 & 3633 & 25.7 \\
\hline 2014 & $18,436,908$ & 13,021 & 86.4 & 8360 & 55.3 & 1029 & 7.2 & 3632 & 23.8 \\
\hline 2015 & $19,116,557$ & 13,974 & 86.6 & 8921 & 55.1 & 1124 & 7.3 & 3929 & 24.2 \\
\hline
\end{tabular}


Table 7 Hispanic (age-adjusted)

\begin{tabular}{|c|c|c|c|c|c|c|c|c|c|}
\hline \multirow[b]{2}{*}{ Year } & \multirow[b]{2}{*}{ ( $n=$ Population) } & \multicolumn{2}{|l|}{ Heart disease } & \multicolumn{2}{|l|}{ Ischemic HD } & \multicolumn{2}{|l|}{ Heart failure } & \multicolumn{2}{|l|}{ All other HD } \\
\hline & & ( $\mathrm{n}=$ deaths) & AAMR & ( $\mathrm{n}=$ deaths) & AAMR & ( $\mathrm{n}=$ deaths) & AAMR & ( $\mathrm{n}=$ deaths) & AAMR \\
\hline 2000 & $35,305,818$ & 25,819 & 196 & 19,744 & 153.2 & 1270 & 10.9 & 4805 & 31.9 \\
\hline 2001 & $37,144,096$ & 27,090 & 193.7 & 20,664 & 151.1 & 1364 & 10.8 & 5062 & 31.9 \\
\hline 2002 & $38,617,620$ & 27,887 & 188.8 & 20,941 & 144.7 & 1412 & 10.8 & 5534 & 33.2 \\
\hline 2003 & $40,049,429$ & 28,298 & 182.1 & 20,783 & 136.8 & 1606 & 11.5 & 5909 & 33.8 \\
\hline 2004 & $41,501,375$ & 27,788 & 169.1 & 20,482 & 127.4 & 1545 & 10.5 & 5761 & 31.2 \\
\hline 2005 & $43,023,614$ & 29,555 & 170.4 & 21,774 & 127.9 & 1721 & 11.3 & 6060 & 31.3 \\
\hline 2006 & $44,606,305$ & 28,921 & 157.8 & 20,939 & 116.4 & 1830 & 11.3 & 6152 & 30.1 \\
\hline 2007 & $46,196,853$ & 29,021 & 149.5 & 20,452 & 107.5 & 1890 & 10.9 & 6679 & 31.1 \\
\hline 2008 & $47,793,785$ & 28,951 & 141.4 & 20,261 & 100.8 & 1966 & 10.7 & 6724 & 30.0 \\
\hline 2009 & $49,327,489$ & 29,611 & 135.8 & 20,228 & 94.7 & 2013 & 10.2 & 7370 & 30.9 \\
\hline 2010 & $50,477,594$ & 30,006 & 132.8 & 20,494 & 92.3 & 2024 & 10 & 7488 & 30.6 \\
\hline 2011 & $52,045,277$ & 30,385 & 123.9 & 20,326 & 84.2 & 2233 & 10.1 & 7826 & 29.6 \\
\hline 2012 & $53,027,708$ & 31,595 & 122 & 20,751 & 81.1 & 2404 & 10.2 & 8440 & 30.7 \\
\hline 2013 & $54,071,370$ & 33,243 & 121.2 & 21,788 & 80.3 & 2544 & 10.1 & 8911 & 30.7 \\
\hline 2014 & $55,387,539$ & 34,021 & 116 & 21,871 & 75.3 & 2742 & 10.2 & 9408 & 30.5 \\
\hline 2015 & $56,592,793$ & 36,401 & 116.9 & 23,055 & 74.5 & 3239 & 11.3 & 10,107 & 31.9 \\
\hline
\end{tabular}

Age-adjusted mortalilty rate per 100,000 person-years, directly standardized to the 2000 U.S. population

Table 8 Non-Hispanic Black (age-adjusted)

\begin{tabular}{|c|c|c|c|c|c|c|c|c|c|}
\hline \multicolumn{10}{|c|}{ Trends in mortality in United States from 2000 to 2015 by gender and race-ethnicity } \\
\hline \multirow[b]{2}{*}{ Year } & \multirow[b]{2}{*}{ ( $n=$ Population) } & \multicolumn{2}{|l|}{ Heart disease } & \multicolumn{2}{|l|}{ Ischemic HD } & \multicolumn{2}{|l|}{ Heart failure } & \multicolumn{2}{|l|}{ All other HD } \\
\hline & & $(n=$ deaths $)$ & AAMR & $(\mathrm{n}=$ deaths $)$ & AAMR & $(\mathrm{n}=$ deaths $)$ & AAMR & $(n=$ deaths $)$ & AAMR \\
\hline 2000 & $35,091,809$ & 76,706 & 328.4 & 50,659 & 220.4 & 4936 & 22.4 & 21,111 & 85.7 \\
\hline 2001 & $35,638,389$ & 76,794 & 322.6 & 50,295 & 215.0 & 5094 & 22.7 & 21,405 & 84.9 \\
\hline 2002 & $36,049,904$ & 76,694 & 317.1 & 49,522 & 208.5 & 5143 & 22.7 & 22,029 & 85.9 \\
\hline 2003 & $36,422,205$ & 76,452 & 309.6 & 48,617 & 200.8 & 5294 & 22.9 & 22,541 & 85.9 \\
\hline 2004 & $36,848,991$ & 73,373 & 290.9 & 46,064 & 186.0 & 5198 & 22.2 & 22,111 & 82.8 \\
\hline 2005 & $37,270,736$ & 73,302 & 282.4 & 45,435 & 178.1 & 5570 & 23 & 22,297 & 81.3 \\
\hline 2006 & $37,719,495$ & 71,461 & 268.2 & 43,992 & 168.0 & 5524 & 22.2 & 21,945 & 78.1 \\
\hline 2007 & $38,184,699$ & 70,443 & 257.4 & 42,152 & 156.5 & 5464 & 21.4 & 22,827 & 79.4 \\
\hline 2008 & $38,651,733$ & 69,918 & 248.1 & 41,373 & 149.4 & 5415 & 20.6 & 23,130 & 78.2 \\
\hline 2009 & $39,104,815$ & 68,811 & 236.4 & 39,956 & 139.8 & 5290 & 19.3 & 23,565 & 77.3 \\
\hline 2010 & $39,437,133$ & 68,215 & 229.5 & 39,047 & 133.4 & 5497 & 19.8 & 23,671 & 76.2 \\
\hline 2011 & $39,944,896$ & 67,595 & 219.3 & 38,928 & 127.9 & 5492 & 19.1 & 23,175 & 72.1 \\
\hline 2012 & $40,391,388$ & 69,147 & 216.3 & 39,005 & 123.4 & 5879 & 19.6 & 24,263 & 73.3 \\
\hline 2013 & $40,802,086$ & 71,102 & 215.5 & 39,199 & 119.9 & 6518 & 21 & 25,385 & 74.7 \\
\hline 2014 & $41,316,519$ & 71,894 & 210.8 & 38,843 & 114.8 & 6962 & 21.6 & 26,089 & 74.4 \\
\hline 2015 & $41,777,483$ & 74,093 & 210.1 & 39,054 & 111.3 & 7772 & 23.3 & 27,267 & 75.5 \\
\hline
\end{tabular}


Table 9 Non-Hispanic American Indian/Alaskan Native (age-adjusted)

\begin{tabular}{|c|c|c|c|c|c|c|c|c|c|}
\hline \multirow[b]{2}{*}{ Year } & \multirow[b]{2}{*}{ ( $\mathrm{n}=$ Population) } & \multicolumn{2}{|l|}{ Heart disease } & \multicolumn{2}{|l|}{ Ischemic HD } & \multicolumn{2}{|l|}{ Heart failure } & \multicolumn{2}{|l|}{ All other HD } \\
\hline & & ( $\mathrm{n}=$ deaths) & AAMR & ( $\mathrm{n}=$ deaths) & AAMR & ( $\mathrm{n}=$ deaths) & AAMR & ( $\mathrm{n}=$ deaths $)$ & AAMR \\
\hline 2000 & $2,344,042$ & 2350 & 197.8 & 1688 & 142.7 & 171 & 16.7 & 491 & 38.5 \\
\hline 2001 & $2,360,621$ & 2353 & 190.6 & 1672 & 135.6 & 163 & 15.9 & 518 & 39.1 \\
\hline 2002 & 2,383,303 & 2421 & 195.7 & 1744 & 141.3 & 182 & 16.8 & 495 & 37.6 \\
\hline 2003 & $2,404,476$ & 2634 & 201.6 & 1855 & 143.5 & 176 & 16.0 & 603 & 42.2 \\
\hline 2004 & $2,428,499$ & 2524 & 192.8 & 1795 & 138.9 & 187 & 16.6 & 542 & 37.2 \\
\hline 2005 & $2,452,970$ & 2576 & 185.7 & 1738 & 126.0 & 216 & 18.3 & 622 & 41.4 \\
\hline 2006 & $2,477,720$ & 2630 & 182.7 & 1810 & 127.6 & 208 & 16.6 & 612 & 38.4 \\
\hline 2007 & $2,502,533$ & 2557 & 171.6 & 1719 & 117.0 & 180 & 14.1 & 658 & 40.6 \\
\hline 2008 & $2,528,470$ & 2549 & 163.6 & 1671 & 108.0 & 230 & 17.4 & 648 & 38.2 \\
\hline 2009 & $2,552,151$ & 2654 & 164.2 & 1737 & 107.8 & 230 & 16.5 & 687 & 39.9 \\
\hline 2010 & $2,569,567$ & 2656 & 161.6 & 1747 & 106.3 & 217 & 16.0 & 692 & 39.3 \\
\hline 2011 & $2,598,792$ & 2805 & 161 & 1836 & 104.8 & 222 & 14.9 & 747 & 41.3 \\
\hline 2012 & $2,620,501$ & 2823 & 153.7 & 1878 & 101.7 & 201 & 12.7 & 744 & 39.3 \\
\hline 2013 & $2,643,000$ & 3002 & 155.5 & 1949 & 100.4 & 230 & 13.8 & 823 & 41.2 \\
\hline 2014 & $2,667,297$ & 3118 & 153.3 & 2009 & 97.8 & 233 & 13.4 & 876 & 42.2 \\
\hline 2015 & $2,689,706$ & 3303 & 154.9 & 2044 & 95.2 & 286 & 15.0 & 973 & 44.8 \\
\hline
\end{tabular}

Age-adjusted mortalilty rate per 100,000 person-years, directly standardized to the 2000 U.S. population

follow-up showing continued decline through 2014 [12]. On the other hand, the prevalence of HF is on the rise [13]. The mortality trends for ischemic heart disease and HF since 2011 parallel these findings and are therefore plausible.

Table 10 Crude mortality rate, total population rates (per 100,000 person years)

\begin{tabular}{lllll}
\hline & Heart disease & Ischemic HD & Heart failure & All other HD \\
\hline 2000 & 252.6 & 183.1 & 19.8 & 49.7 \\
2001 & 245.7 & 176.2 & 20.0 & 49.5 \\
2002 & 242.3 & 171.9 & 19.6 & 50.8 \\
2003 & 236.1 & 165.5 & 19.8 & 50.9 \\
2004 & 222.8 & 154.1 & 19.5 & 49.2 \\
2005 & 220.7 & 150.8 & 19.9 & 49.9 \\
2006 & 211.7 & 142.6 & 20.2 & 48.9 \\
2007 & 204.5 & 134.9 & 18.8 & 50.8 \\
2008 & 202.8 & 133.3 & 18.7 & 50.9 \\
2009 & 195.4 & 125.9 & 18.4 & 51.1 \\
2010 & 193.6 & 122.9 & 18.7 & 51.9 \\
2011 & 191.5 & 120.4 & 18.7 & 52.3 \\
2012 & 191.0 & 118.3 & 19.2 & 53.5 \\
2013 & 193.3 & 117.1 & 20.6 & 55.6 \\
2014 & 192.7 & 114.3 & 21.5 & 56.8 \\
2015 & 197.2 & 114.1 & 23.4 & 59.7 \\
\hline
\end{tabular}

CVD remain a major cause of health loss internationally. Per the recent GBD (Global Burden of Disease) study, although dramatic declines in CVD occurred in regions with high socioeconomic status, only a gradual decrease or no change was noted in most other regions [14]. Of note, the data analyzed in our study used common groupings of ICD-10 codes to define heart disease and its subtypes such as IHD and all other HD in National Vital Statistics reports for the U.S. [15] that may be slightly different than codes used in GBD studies to define CVD and subtypes [16]. Therefore, the mortality numbers may vary. Similarly, in another study, trends in CHD and CVD mortality continue to be less favorable in Latin America than in Canada and in the U.S. [17].

The National Center for Health Statistics recently reported that deaths considered HF-related (i.e., HF reported anywhere on the death certification) declined from 2000 to 2012 but increased from 2012 to 2014 [18]. It is possible that HF is being inappropriately designated as the underlying cause of death in many instances [19]. This report noted that IHD was the underlying cause of death in 2014 for $23.9 \%$ of HF-related deaths in adults aged 45 years and older but did not report on the frequency of IHD as a listed cause of death when HF was recorded as the underlying cause of death. This might slightly attenuate the downward trend in the IHD mortality rate if $\mathrm{HF}$ is being designated as the underlying cause of death when it is due to IHD. 
Another potential cause of misclassification of HFrelated mortality is competing mortality with a nonCVD cause. While it is possible that declining cancer rates could result in the recent increasing trend in HF mortality and this year's increase in HD mortality, it is unlikely since cancer mortality has been declining at a fairly stable rate of $1.5 \%$ per year since 2000 [1]. The most plausible sources for competing non-CVD mortality are diabetes (E10-E14) and chronic lower respiratory diseases (J40-47) which have declined minimally from 2011 to 2015 (data not shown).

It is well-recognized that HF is a major and growing public health problem. Earlier estimates from projection models for the U.S. suggest that the prevalence of HF will increase by $46 \%$ from 2012 to 2030 [13]. It has been suggested that the absence of a national surveillance system significantly impedes the ability to track and manage this expected increase in HF [20]. Given this, present CDC mortality data becomes an important indicator for burden of HF. Another matter of importance is a rising proportion of patients having HF with preserved ejection fraction (HFpEF), accounting for more than $50 \%$ of incident HF cases, and no definitive treatment to so far, has been proven effective in reducing the morbidity and mortality of HFpEF [21]. Further concomitant multiple comorbid conditions are frequent in this patient population, [22] with a recent analysis from Denmark showing an increasing prevalence of comorbidities, including diabetes mellitus and hypertension, especially in younger patients with HF [23]. It is plausible that the increasing prevalence of these comorbidities and lower death rates after acute myocardial infarction are contributing to increased HF-related mortality rates. Whereas better risk factor control strategies to prevent HF may reduce the incidence, [24] more effective treatments for patients with established HF would be expected to reduce case-fatality.

\section{Conclusions}

While the mortality rate attributed to HD slowed substantially between 2011 and 2014 nationally before turning upward in 2015, trajectories among HD subgroups were heterogeneous, with IHD-related death continuing to decline while death attributed to HF and other causes of HD increased. While systematic efforts to prevent and treat IHD appear to be effective and require continued vigilance, an expanded focus on strategies to reduce deaths from HF and those attributed to other HD conditions appear needed. Finally, addressing the complex care of HF patients with multiple morbidities would likely need system-wide, multipronged health care interventions, with particularly urgent attention to developing more effective treatments for HFpEF [25].

\section{Abbreviations}

AAMR: Age-adjusted mortality rate; CVD: Cardiovascular disease; HD: Heart disease; HF: Heart failure; ICD-10: International Statistic Classification of Diseases and Related Health Problems, Tenth Edition; IHD: Ischemic heart disease

\section{Acknowledgements \\ Karin M. Winter for administrative and technical support}

\section{Funding}

Funding for this work was provided by the Cardiovascular Research Network (CVRN) with funding from the National Heart Lung and Blood Institute (NHLBI) (U19 HL91179-01) and the American Recovery and Reinvestment Act of 2009 (RC2 HL101666) (Sidney, Go)

\section{Availability of data and materials}

The source of data for determining all mortality rates for the study was the U.S. Centers for Disease Control and Prevention's Wide-Ranging Online Data for Epidemiologic Research (CDC WONDER) dataset. CDC WONDER is a menu-driven system that makes the information resources of the Centers for Disease Control and Prevention (CDC) available to public health professionals and the public at large. For this study, the "Underlying Cause of Death, 1999-2015" section of CDC WONDER was accessed. For each cause of death noted in the paper, we entered an inquiry through the menu driven system for the number of deaths, crude death rate, and age-adjusted death rate for each of the years, 2000-2015. The link to the CDC "Underlying Cause of Death, 1999-2015" data system is https://wonder.cdc.gov/ucd-icd10.html.

\section{Authors' contributions}

Dr. Sidney had full access to all of the data in the study and takes responsibility for the integrity of the data and the of the data analysis. Study concept and design: SS, JSR. Acquisition of data: SS, JSR. Analysis and interpretation of data: All authors. Drafting of the manuscript: SS, JSR. Critical revision of the manuscript: All authors. Statistical analysis: SS, CPQ, MES. Obtained funding: SS, ASG. Administrative, technical, or material support: SS. Study supervision: SS, JSR. All authors read and approved the final manuscript.

Ethics approval and consent to participate

Not applicable.

\section{Consent for publication}

Not applicable.

\section{Competing interests}

Relevant financial activities outside the submitted work included research grant support related to cardiovascular disease for Dr. Go from Astra-Zeneca, Sanofi, and CSL Behring. Dr. Rana reports receiving grant funding from Regeneron and Sanofi to his institution.

\section{Publisher's Note}

Springer Nature remains neutral with regard to jurisdictional claims in published maps and institutional affiliations.

\section{Author details}

${ }^{1}$ Division of Research, Kaiser Permanente Northern California, 2000 Broadway, Oakland, CA 94612, USA. ²Department of Endocrinology, Kaiser Permanente South San Francisco Medical Center, South San Francisco, CA, USA. ${ }^{3}$ Departments of Epidemiology, Biostatistics and Medicine, University of California, San Francisco, San Francisco, CA, USA. ${ }^{4}$ Department of Health Research and Policy, Stanford University School of Medicine, Stanford, CA, USA. ${ }^{5}$ Department of Cardiology, Kaiser Permanente Oakland Medical Center, Oakland, CA, USA. ${ }^{6}$ Department of Medicine, University of California, San Francisco, San Francisco, CA, USA.

Received: 24 March 2017 Accepted: 12 July 2017

Published online: 18 July 2017

\section{References}

1. Sidney S, Quesenberry CP, Jr, Jaffe MG, Sorel M, Nguyen-Huynh MN Kushi LH, Go AS, Rana JS. Recent trends in cardiovascular mortality in 
the United States and public health goals. JAMA Cardiol. 2016. doi:10.1001/ jamacardio.2016.1326

2. Xu JQ, Murphy SL, Kochanek KD, Arias E. Mortality in the United States, 2015. NCHS data brief, no 267. Hyattsville, MD: National Center for Health Statistics. 2016.

3. Center for Disease Control and Prevention. Underlying cause of death, 1999-2014 request. http://wonder.cdc.gov/ucd-icd10.html. Accessed 4 Aug 2016.

4. Klein RJ, Schoenberg CA. Age adjustment using the 2000 projected U.S. population. Healthy People Statistical Notes, no. 20. Hyattsville, Maryland: National Center for Health Statistics. January 2001. https://www.cdc.gov/ nchs/data/statnt/statnt20.pdf.

5. Navar AM, Peterson ED, Wojdyla D, Sanchez RJ, Sniderman AD, D'Agostino RB Sr, Pencina MJ. Temporal changes in the association between modifiable risk factors and coronary heart disease incidence. JAMA. 2016;316(19):2041-3.

6. Rates of Diagnosed Diabetes per 100 Civilian, Non-Institutionalized Population, by Age, United States, 1980-2014. https://www.cdc.gov/ diabetes/statistics/prev/national/figbyage.htm. Accessed 17 Dec 2016.

7. Rana JS, Liu JY, Moffet HH, Jaffe M, Karter AJ. Diabetes and prior coronary heart disease are not necessarily risk equivalent for future coronary heart disease events. J Gen Intern Med. 2016;31(4):387-93.

8. Yeh RW, Sidney S, Chandra M, Sorel M, Selby JV, Go AS. Population trends in the incidence and outcomes of acute myocardial infarction. N Engl J Med. 2010;362(23):2155-65.

9. Gupta A, Wang Y, Spertus JA, Geda M, Lorenze N, Nkonde-Price C, D'Onofrio G, Lichtman JH, Krumholz HM. Trends in acute myocardial infarction in young patients and differences by sex and race, 2001 to 2010. J Am Coll Cardiol. 2014;64:337-45.

10. Krumholz HM, Normand SL, Wang Y. Trends in hospitalizations and outcomes for acute cardiovascular disease and stroke, 1999-2011. Circulation. 2014:130:966-75

11. Reynolds K, Go AS, Leong TK, Boudreau DM, Cassidy-Bushrow AE, Fortmann SP, Goldberg RJ, Gurwitz JH, Magid DJ, Margolis KL, McNeal CJ, Newton KM, Novotny R, Quesenberry CP Jr, Rosamond WD, Smith DH, VanWormer JJ, Vupputuri S, Waring SC, Williams MS, Sidney S. Trends in incidence of hospitalized acute myocardial infarction in the cardiovascular research network (CVRN). Am J Med. 2017;130(3):317-27.

12. Solomon MD, Leong TK, Rana JS, Xu Y, Go AS. Community-based trends in acute myocardial infarction from 2008 to 2014. J Am Coll Cardiol. 2016;68(6):666-8. doi:10.1016/j.jacc.2016.03.607.

13. Heidenreich PA, Albert NM, Allen LA, Bluemke DA, Butler J, Fonarow GC, Ikonomidis JS, Khavjou O, Konstam MA, Maddox TM, Nichol G, Pham M, Piña IL, Trogdon JG. Forecasting the impact of heart failure in the United States: a policy statement from the American Heart Association. Circ Heart Fail. 2013;6(3):606-19.

14. Roth GA, Johnson C, Abajobir A, Abd-Allah F, Abera SF, Abyu G, et al. Global, Regional, and National Burden of Cardiovascular Diseases for 10 Causes, 1990 to 2015. J Am Coll Cardiol. 2017. doi:10.1016/j.jacc.2017.04.052. [Epub ahead of print] PubMed PMID: 28527533.

15. Kochanek KD, Murphy SL, Xu JQ, Tejada-Vera B. Deaths: Final data for 2014 National vital statistics reports; vol 65 no 4. Hyattsville, MD: National Center for Health Statistics. 2016.

16. $\mathrm{WHO}$ methods and data sources for global burden of disease estimates 2000-2015. Global Health Estimates Technical Paper WHO/HIS/IER/GHE/ 2017.1 Department of Information, Evidence and Research, World Health Organization, Geneva. January 2017.

17. Pagan E, Chatenoud L, Rodriguez T, Bosetti C, Levi F, Malvezzi M, La Vecchia C, Negri E, Bertuccio P. Comparison of trends in mortality from coronary heart and cerebrovascular diseases in north and South America: 1980 to 2013. Am J Cardiol. 2017;119(6):862-71

18. $\mathrm{Ni} \mathrm{H}, \mathrm{Xu} J \mathrm{Q}$, Recent trends in heart failure-related mortality. United States, 2000-2014. NCHS data brief, no 213. Hyattsville, MD: National Center for Health Statistics, 2015.

19. Snyder ML, Love SA, Sorlie PD, Rosamond WD, Antini C, Metcalf PA, Hardy S, Suchindran CM, Shahar E, Heiss G. Redistribution of heart failure as the cause of death: the atherosclerosis risk in communities study. Popul Health Metr. 2014:12(1):10. doi:10.1186/1478-7954-12-10.

20. Rosamond WD, Johnson A. Trends in heart failure incidence in the community: a gathering storm. Circulation. 2017:135(13):1224-6.
21. Oren O, Goldberg S. Heart failure with preserved ejection fraction: diagnosis and management. Am J Med. 2017;130(5):510-6.

22. Dunlay SM, Roger VL. Understanding the epidemic of heart failure: past, present, and future. Curr Heart Fail Rep. 2014;11(4):404-15.

23. Christiansen MN, Køber $L$, Weeke $P$, et al. Age-specific trends in incidence, mortality and comorbidities of heart failure in Denmark, 1995 to 2012. Circulation. 2017:135:1214-23.

24. Ahmad FS, Ning H, Rich JD, Yancy CW, Lloyd-Jones DM, Wilkins JT. Hypertension, obesity, diabetes, and heart failure-free survival: the cardiovascular disease lifetime risk pooling project. JACC Heart Fail. 2016:4(12):911-9.

25. Nanayakkara S, Kaye DM. Management of heart failure with preserved ejection fraction: a review. Clin Ther. 2015;37(10):2186-98.

\section{Submit your next manuscript to BioMed Central and we will help you at every step:}

- We accept pre-submission inquiries

- Our selector tool helps you to find the most relevant journal

- We provide round the clock customer support

- Convenient online submission

- Thorough peer review

- Inclusion in PubMed and all major indexing services

- Maximum visibility for your research

Submit your manuscript at www.biomedcentral.com/submit 